\title{
Deformation of Uniruled Manifolds
}

By

\author{
Akira FujIKI*
}

Let $f: X \rightarrow S$ be a proper smooth morphism of complex spaces with connected fibers. Suppose that each point $s \in S$ has a neighborhood $s \in U$ such that there exist a Kähler manifold $Y_{U}$, a proper morphism $g: Y_{U} \rightarrow U$ and a generically surjective meromorphic map $h: Y_{U} \rightarrow X_{U}$ over $U$. The condition is satisfied, e.g., if each fiber of $f$ is Moishezon or if $f$ is a Kähler morphism in the sense of [1]. Then the main purpose of this note is to show the following (Proposition 2.3): Under the above condition if $X_{o}$ is uniruled for some $o \in S$, then $X_{s}$ is uniruled for all $s \in S$. (See Theorem 2.4 for a little more general statement.) Thus the class of uniruled manifolds are closed under smooth deformations which is 'weakly polarized' in the sense of the above condition. The method of proof is nothing but a simple adaptation to our situation of the method used by Mori in [8] (cf. Lemma 2.6).

Let $R$ be the set of those points $s \in S$ for which $X_{s}$ are ruled. Then we also show that, when restricted to any relatively compact subdomain $S^{\prime}$ of $S, R$ is a union of at most countably many analytic subsets of $S^{\prime}$, under the stronger assumption that $f$ is a Moishezon morphism (Proposition 3.3).

Proposition 2.3 plays an important role in our construction of the coarse moduli space for the polarized family of nonuniruled compact Kähler manifolds in [5].

Convention. In this paper complex spaces are in general assumed to be reduced. Let $f: X \rightarrow S$ be a morphism of complex spaces. For any locally closed analytic subspace $V$ of $S$ we set $X_{V}=X \times{ }_{S} V$ and $f_{V}=\left.f\right|_{X_{V}}: X_{V} \rightarrow V$. If $V$ consists of a single point $s \in S$ we write $X_{s}$ instead of $X_{\{s\}}$. For $X=X_{\alpha}$ we often writc $X_{\alpha, s}$ instead of $\left(X_{\alpha}\right)_{s}$. Pr denotes the complex projective space of complex dimension $r$.

Received October 25, 1980.

* Research Institute for Mathematical Sciences, Kyoto University. Current address: Yoshida College, Kyoto University, Kyoto 606, Japan. 


\section{§1. Preliminaries}

The purpose of this section is to fix notations and to summarize some known results on the Douady space of a complex space. The results stated in this section will often be used without further reference in the later sections.

a) Let $h: Y \rightarrow S$ be a morphism of complex spaces. Then we denote by $\beta_{Y / S}: D_{Y / S} \rightarrow S$ the relative Douady space of $Y$ over $S$, parametrizing the compact analytic subspaces of $X$ contained in the fibers of $h$ (cf. [1]). Let $\rho_{Y / S}$ : $Z_{Y / S} \rightarrow D_{Y / S}$ be the corresponding universal family, so that there is a natural embedding $Z_{Y / S} \leqq D_{Y / S} \times{ }_{S} Y$ with $\rho_{Y / S}$ induced by the natural projection $D_{Y / S} \times{ }_{S} Y$ $\rightarrow D_{Y / S}$. Recall that $\rho_{Y / S}$ is proper and flat. We denote by $\pi_{Y / S}$ the natural morphism $Z_{Y / S} \rightarrow Y$ induced by the other projection $D_{Y / S} \times{ }_{S} Y \rightarrow Y$. Let $D_{\alpha}$ be any irreducible component of $D_{Y / S \text {, red }}$, the underlying reduced subspace of $D_{Y / S}$. Then we denote by $\rho_{\alpha}: Z_{\alpha} \rightarrow D_{\alpha}$ the restriction of $\rho_{Y / S}$ over $D_{\alpha}$ and by $\pi_{\alpha}: Z_{\alpha} \rightarrow Y$ the restriction of $\pi_{Y / S}$ to $Z_{\alpha}$. Thus we have the following commutative diagram

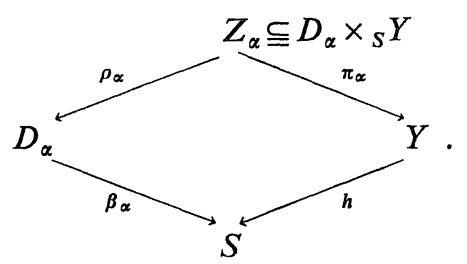

When $S$ is a point, we shall write $\rho_{Y}: Z_{Y} \rightarrow D_{Y}$ and $\pi_{Y}: Z_{Y} \rightarrow Y$ instead of $\rho_{Y / S}$ and $\pi_{Y / S}$ respectively.

In general for any $s \in S, D_{Y / S, s}$ is naturally isomorphic to $D_{Y_{s}}$, and we often identify these two spaces.

Let $f: X \rightarrow S$ be a morphism of complex spaces. Let $Y=\boldsymbol{P}^{1} \times X$ and $h=p f: Y \rightarrow S$ where $p: Y \rightarrow X$ is the natural projection. Obviously we have $Y_{s}=\boldsymbol{P}^{1} \times X_{s}$ for all $s \in S$. We set $\bar{\pi}_{Y / S}=p \pi_{Y / S}: Z_{Y / S} \rightarrow X$ and $\varphi_{Y / S}=q \pi_{Y / S}: Z_{Y / S}$ $\rightarrow \boldsymbol{P}^{1}$ where $q: Y \rightarrow \boldsymbol{P}^{1}$ is the natural projection. Let $D_{\alpha}$ be any irreducible component of $D_{Y / S \text {, red }}$ as above. Then we also set $\bar{\pi}_{\alpha}=p \pi_{\alpha}: Z_{\alpha} \rightarrow X$ and $\varphi_{\alpha}$ $=q \pi_{\alpha}: Z_{\alpha} \rightarrow \boldsymbol{P}^{1}$.

b) Let $X$ be a compact complex space and $Y=\boldsymbol{P}^{1} \times X$. Let $H=\operatorname{Hol}\left(\boldsymbol{P}^{1}\right.$, $X$ ) be the set of all holomorphic maps $h: \boldsymbol{P}^{1} \rightarrow X$ of $\boldsymbol{P}^{1}$ into $X$. Associating to each $h$ its graph $\Gamma_{h} \subseteq \boldsymbol{P}^{1} \times X$ we may regard $H$ as a subspace of the Douady space 
$D_{Y}$ of $Y$. In fact, it is known that $H$ is Zariski open in $D_{Y}$ with respect to this inclusion. Let $H_{0}$ be the subset of $H$ consisting of non-constant holomorphic maps. Then $H_{0}$ is again Zariski open in $D_{Y}$. Then a point $d \in D_{Y}$ belongs to $H_{0}$ if and only if the induced map $\varphi_{Y, d}: Z_{Y, d} \rightarrow \mathbb{P}^{1}$ is isomorphic and $\operatorname{dim} \pi_{Y}\left(Z_{Y, d}\right)$ $=1$ in $X$, where $Z_{Y, d}=\rho_{Y}^{-1}(d)$. This immediately follows from the definition. Let $B$ be an irreducible analytic subspace of $D_{Y \text {.red. }}$ Let $\pi_{B}: Z_{B} \rightarrow B$ and $\varphi_{B}: Z_{B}$ $\rightarrow \boldsymbol{P}^{1}$ be induced by $\pi_{Y}$ and $\varphi_{Y}$ respectively. Let $U_{B}=B \cap H_{0}$. Then it follows that if $U_{B} \neq \emptyset, Z_{B}$ is reduced and irreducible and $\rho_{B} \times \varphi_{B}: Z_{B} \rightarrow B \times \mathbb{P}^{1}$ is a bimeromorphic morphism which is isomorphic over $U \times \mathbb{P}^{1}$.

c) Let $h: Y \rightarrow S$ and $\beta=\beta_{Y / S}: D_{Y / S} \rightarrow S$ be as in a). Let $d \in D_{Y / S}$ and $o$ $=\beta(d) \in S$. Let $Z_{d}=Z_{Y / S, d}=\rho_{Y / S}^{-1}(d)$. Consider $Z_{d}$ naturally as a subspace of $Y_{o}$ by $\pi_{Y / S, o}$. Suppose that $h$ is smooth and $Z_{d}$ is smooth. Let $N=N_{Z_{d} / Y_{o}}$ be the normal sheaf of $Z_{d}$ in $Y_{o}$. Then we recall the following [7]: $\beta$ is smooth at $d$ if $H^{1}\left(Z_{d}, N\right)=0$.

Let $Y=\mathbb{P}^{1} \times X$ as in b) with $X$ nonsingular. Identifying $D_{Y / S, o}$ with $D_{Y_{o}}$ as in a), consider $d \in D_{Y_{o}}$. Then suppose that $d \in H=\operatorname{Hol}\left(\mathbb{P}^{1}, X_{o}\right) \subseteq D_{Y_{o}}$ in the notation of b) (with $X=X_{o}$ ) so that $d$ corresponds to a holomorphic map $h_{d}$ : $\mathbb{P}^{1}$ $\rightarrow X_{o}$. Then $Z_{d} \cong \Gamma_{h} \cong P^{1}$ and we have $N_{Z_{d} / Y_{o}} \cong h_{d}^{*} \Theta_{X_{o}}$ where $\Theta_{X_{o}}$ is the sheaf of germs of holomorphic vector fields on $X_{o}$. Thus we obtain the following: $\beta$ is smooth at $d \in H_{0}$ if $H^{1}\left(\mathbb{P}^{1}, h_{d}^{*} \Theta_{X_{0}}\right)=0$.

d) Let $f: X \rightarrow S$ be a proper morphism of complex spaces. Then we shall write $f \in \mathscr{C} / S$ if there exist a proper and locally Kähler morphism $g: Y \rightarrow S$ (e.g. this is the case when $Y$ is a Kähler manifold) (cf. [3, Section 2]), and a generically surjective meromorphic $S$-map $h: Y \rightarrow X$. For instance $f \in \mathscr{C} / S$ if $f$ itself is a proper Kähler morphism [1], or if $f$ is a Moishezon morphism, i.e., bimeromorphic over $S$ to a projective morphism. Note that in the latter case each fiber of $f$ is a Moishezon space. Conversely, if $f$ is smooth and each fiber is Moishezon, then for every $s \in S$ there exists a neighborhood $s \in U$ such that $f_{U}$ is Moishezon in the sense mentioned above (cf. [6]). We write $f \in \operatorname{loc}-\mathscr{C} / S$ if for every $s \in S$ there exists a neighborhood $s \in U$ such that the induced morphism $f_{U}: X_{U} \rightarrow U \in \mathscr{C} / S$. Let $X$ be a compact complex space. Then we write $X \in \mathscr{C}$ if there exist a compact Kähler manifold $Y$ and a surjective meromorphic map $h: Y \rightarrow X$. If $f \in \mathscr{C} / S$, then each fiber $X_{s}$ of $f$ belongs to $\mathscr{C}$.

Recall from [1] [2] the following facts which explains our assumptions on $f$ in the results below. If $f \in \mathscr{C} / S$, then for any relatively compact subdomain 
$S^{\prime}$ of $S$ and for any irreducible component $D_{\alpha}$ of $D_{X^{\prime} / S^{\prime} \text {,red }}$ such that $Z_{\alpha}$ is reduced, the natural morphism $D_{\alpha} \rightarrow S^{\prime}$ is proper and belongs to $\mathscr{C} / S^{\prime}$, where $X^{\prime}=f^{-1}\left(S^{\prime}\right)$. Similarly if $f$ is Moishezon, then $D_{\alpha} \rightarrow S^{\prime}$ is again proper and Moishezon.

\section{§2. Deformations of Uniruled Manifolds}

Let $N$ be a complex manifold and $\varepsilon: E \rightarrow N$ a holomorpihc vector bundle of finite rank $r$. Identifying $N$ with the zero section of $E$ we set $\boldsymbol{P}(E)=(E-N) / \boldsymbol{C}^{*}$, $C^{*}$ acting naturally on each fiber of $\varepsilon$. Then $\varepsilon$ induces a natural projection $\bar{\varepsilon}: \boldsymbol{P}(E) \rightarrow N$ making $\boldsymbol{P}(E)$ a holomorphic fiber bundle over $N$ with typical fiber $\boldsymbol{P}^{r-1}$.

Definition 2.1. Let $X$ be an irreducible compact complex space. 1) $X$ is called ruled if there exist a compact complex manifold $N$ and a holomorphic vector bundle $E$ of rank 2 over $N$ as above such that $X$ is bimeromorphic to $\boldsymbol{P}(E) .2) X$ is called uniruled if there exist $N$ and $E$ as above such that there exists a generically surjective meromorphic map $\lambda: \mathbb{P}(E) \rightarrow X$ which is not factored by $\bar{\varepsilon}$ and a meromorphic map $N \rightarrow X$.

Remark. If $X$ is Moishezon and uniruled, then we can take the above $\lambda$ to be generically finite.

Let $X$ be a compact complex space. Let $g: Z \rightarrow T$ and $\psi: Z \rightarrow X$ be morphisms of compact irreducible complex spaces. Then we call the pair $(g: Z \rightarrow T$, $\psi: Z \rightarrow X)$ a covering family of rational curves on $X$ if the following conditions are satisfied;1) $\psi$ is surjective and 2) there exists a Zariski open subset $U \subseteq T$ such that for all $t \in U, Z_{t}$ are isomorphic to $\boldsymbol{P}^{1}$ and $\operatorname{dim} \psi\left(Z_{t}\right)=1$.

Lemma 2.2. Let $X$ be an irreducible compact complex space in $\mathscr{C}$. Then the following conditions are equivalent. 1) $X$ is uniruled. 2) $X$ admits a covering family of rational curves. 3) Let $Y=\mathbb{P}^{1} \times X$. Then there exists an irreducible component $D_{\alpha}$ of $D_{Y, \text { red }}$ such that $D_{\alpha} \cap H_{0} \neq \varnothing(c f$. Section $\left.1, b)\right)$ and that $\bar{\pi}_{\alpha}: Z_{\alpha} \rightarrow X$ is surjective.

Proof. 1) $\rightarrow$ 2). Let $\bar{\varepsilon}: \boldsymbol{P}(E) \rightarrow N$ and $\lambda: \boldsymbol{P}(E) \rightarrow X$ be as in 2) of Definition 2.1. Let $\boldsymbol{P}=\boldsymbol{P}(E)$. Eliminating the indeterminacy of $\lambda$ by some bimeromorphic morphism $\tilde{\boldsymbol{P}} \rightarrow \boldsymbol{P}$, we obtain the induced surjective morphism $\tilde{\lambda}: \tilde{\boldsymbol{P}} \rightarrow X$ and $\tilde{\varepsilon}: \tilde{\boldsymbol{P}} \rightarrow N$. Clearly the general fiber of $\tilde{\varepsilon}$ is isomorphic to $\boldsymbol{P}^{1}$. The pair $(\tilde{\lambda}$ : $\tilde{\boldsymbol{P}} \rightarrow X, \tilde{\varepsilon}: \tilde{\boldsymbol{P}} \rightarrow N$ ) then gives the covering family of rational curves on $X .2$ ) $\rightarrow 3$ ). 
Let $(g: Z \rightarrow T, \psi: Z \rightarrow X)$ be a covering family of rational curves on $X$. Let $U \subseteq T$ be a Zariski open subset such that $g$ is smooth over $U$ and $Z_{t} \cong \mathbb{P}^{1}$ for $t \in U$. Then $g_{U}: Z_{U} \rightarrow U$ is a holomorphic $\mathbb{P}^{1}$-bundle. Let $r: P \rightarrow U$ be the principal $P G L(2)$-bundle to which $g_{U}$ is associated. Then the induced bundle $P \times{ }_{U} Z_{U} \rightarrow P$ is trivial so that we have a $P$-isomorphism $\delta: P \times \mathbb{P}^{1} \cong P \times{ }_{U} Z_{U}$. Let $\mu=\psi p_{2} \delta: P \times \mathbb{P}^{1} \rightarrow X$, where $p_{2}: P \times Z_{U} \rightarrow Z_{U}$ is the natural projection. Since $\psi$ is surjective and hence is smooth at general point of $Z_{U}$, the image of $\mu$ contains an open subset of $X$ and $\operatorname{dim} \mu\left(\{p\} \times \mathbb{P}^{1}\right)=1$ for all $p \in P$.

Let $\Gamma \cong P \times \mathbb{P}^{1} \times X$ be the graph of $\mu$. Let $\rho: \Gamma \rightarrow P, \beta: \Gamma \rightarrow P \times \mathbb{P}^{1}, \tilde{\pi}$ : $\Gamma \rightarrow X$ be the morphisms induced by the natural projections from $P \times \mathbb{P}^{1} \times X$. Clearly $\tilde{\pi}=\mu \cdot \beta$. Further since $\beta$ is an isomorphism over $P(\Gamma$ is over $P$ by $\rho$ ), $\rho$ is naturally regarded as a smooth family of subspaces of $Y=\mathbb{P}^{1} \times X$. Thus there exists a unique morphism $\tau: P \rightarrow D_{Y}$ such that $\rho$ is induced from the universal family $\rho_{Y}: Z_{Y} \rightarrow D_{Y}$ by $\tau$. We have the associated commutative diagram

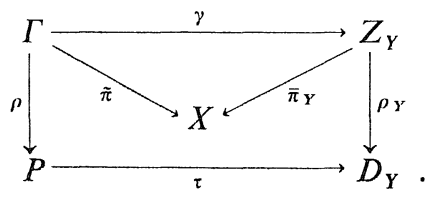

Let $D_{\alpha}$ be any irreducible component of $D_{Y, \text { red }}$ containing $\tau(P)$. Then we claim that this $D_{\alpha}$ enjoys the desired property. Note first that for all $d \in \tau(P)$ we have $Z_{\alpha, d} \cong \Gamma_{p} \cong Z_{p} \cong \mathbb{P}^{1}$ and $\bar{\pi}_{\alpha}\left(Z_{\alpha, d}\right)=\bar{\pi}_{\alpha} \gamma\left(\Gamma_{p}\right)=\tilde{\pi}\left(\Gamma_{p}\right)=\mu\left(Z_{p}\right)$ for any $p \in P$ with $\tau(p)=d$. Hence for such $d, Z_{\alpha, d} \cong \mathbb{P}^{1}$ and $\operatorname{dim} \bar{\pi}_{\alpha}\left(Z_{\alpha, d}\right)=1$, i.e., $D_{\alpha} \cap H_{0} \neq \emptyset$ (cf. Section 1, b)). Moreover we have $\bar{\pi}_{\alpha}\left(Z_{\alpha}\right)=X$. In fact, $Z_{\alpha}$ is compact since $X \in \mathscr{C}$, so that $\bar{\pi}_{\alpha}\left(Z_{\alpha}\right)$ is an analytic set. Further $\bar{\pi}_{\alpha}\left(Z_{\alpha}\right) \supseteqq \tilde{\pi}(\Gamma)=\mu\left(P \times \mathbb{P}^{1}\right)$ and $\mu\left(P \times \mathbb{P}^{1}\right)$ contains an open subset of $X$. Hence $X=\bar{\pi}_{\alpha}\left(Z_{\alpha}\right)$. 3) $\left.\rightarrow 1\right)$ Let $D_{\alpha}$ be as in 3). Then $\rho_{\alpha} \times \varphi_{\alpha}: Z_{\alpha} \rightarrow D_{\alpha} \times \mathbb{P}^{1}$ is bimeromorphic (cf. Section 1, b)). Thus there exists a surjective meromorphic map $\tilde{D}_{\alpha} \times \mathbb{P}^{1} \rightarrow X$ which is not factored by the projection $\widetilde{D}_{\alpha} \times \mathbb{P}^{1} \rightarrow \widetilde{D}_{\alpha}$ where $\widetilde{D}_{\alpha}$ is any resolution of $D_{\alpha}$ Q. E. D.

Remark. If $X$ is uniruled, then the Kodaira dimension $\kappa(X)=-\infty$. Proof: In 3) of the above lemma take any smooth subspace $N \subseteq H_{0} \cap D_{\alpha}$ with $\operatorname{dim} N=\operatorname{dim} X-1$ such that the associated holomorphic map $h_{N}: \mathbb{P}^{1} \times N \rightarrow X$ is locally biholomorphic at some point. Then any non-zero element of $\Gamma\left(X, K_{X}^{m}\right)$, $m>0$, would induce a nonzero element of $\Gamma\left(P^{1} \times N, K_{N}^{m}\right)$ which in turn gives rise to a nonzero element of $\Gamma\left(\boldsymbol{P}_{v}^{1}, K_{\boldsymbol{P}_{v}}^{m_{1}}\right)=0$ for general $v \in N\left(\right.$ where $\left.\mathbb{P}_{v}^{1}=\mathbb{P}^{1} \times v\right)$, leading to a contradiction. 
The following proposition shows the stability of uniruled complex manifolds under smooth deformations which is 'polarized' in a weak sense there.

Proposition 2.3. Let $f: X \rightarrow S$ be a proper smooth morphism of complex spaces with connected fibers. Suppose that $f \in \operatorname{loc}-\mathscr{C} / S$ (cf. Section 1, d)) and that $S$ is connected. Then if $X_{o}$ is uniruled for some ofS, $X_{s}$ are uniruled for all $s \in S$.

For the proof we may clearly assume that $S$ is irreducible. Hence the proposition is a special case of the following:

Theorem 2.4. Let $f: X \rightarrow S$ be a proper morphism of irreducible complex spaces with connected fibers. Suppose that $f \in \operatorname{loc}-\mathscr{C} / S$ (cf. Section 1, d)) and that there exists an open subset $U$ of $S$ such that $\left.f\right|_{f^{-1}(U)}: f^{-1}(U) \rightarrow U$ is smooth. Then if $X_{o}$ is uniruled for some ofU, every irreducible component of $X_{s}$ is again uniruled for all $s \in S$.

First we need a lemma. Consider a commutative diagram

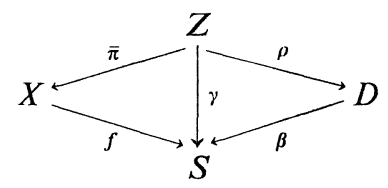

of irreducible complex spaces where $\beta$ and $f$ are proper. Let $o \in S$ be a fixed point. We assume that $\rho$ is proper, flat and surjective and that in a neighborhood of $X_{o}, f$ is smooth and has connected fibers.

Lemma 2.5. Let $d_{o} \in D$ with $\beta\left(d_{o}\right)=o$. Assume the following conditions; 1) $\beta$ is smooth at $d_{o}$, 2) $Z_{d_{o}}=\rho^{-1}\left(d_{o}\right)$ is irreducible, smooth and of dimension 1 and $\operatorname{dim} \bar{\pi}\left(Z_{d_{o}}\right)=1$, and 3) $\bar{\pi}\left(Z_{o}^{\prime}\right)=X_{o}$ for some irreducible component $Z_{o}^{\prime}$ of $Z_{o}$ containing $Z_{d_{o}}$. Then for each $s \in S$ and for each irreducible component $X_{s, k}$ of $X_{s, \mathrm{red}}$ there exists an irreducible component $Z_{s, k}$ of $Z_{s, \text { red }}$ such that $\bar{\pi}\left(Z_{s, k}\right)=X_{s, k}$ and that $\operatorname{dim} \bar{\pi}\left(\left(Z_{s, k}\right)_{d}\right)=1$ for all $d \in D_{s, k}$ where $\left(Z_{s, k}\right)_{d}=Z_{s, k} \cap Z_{d}$ and $D_{s, k}=\rho\left(Z_{s, k}\right)$.

Proof. Note that $\gamma$ and $\bar{\pi}$ also are proper. First we show that $\bar{\pi}$ is surjective. Since $\rho$ is flat and $Z_{d_{o}}$ is smooth, $\rho$ is smooth along $Z_{d_{o}}$. Together with the smoothness of $\beta$ at $d_{o}$, this implies that $\gamma$ is smooth in a neighborhood $V$ of $Z_{d_{o}}$ in $Z$. In particular $\gamma(V)$ is open. Moreover for each $s \in \gamma(V)$ and for each connected component of $Z_{s i}(V)$ of $Z_{s} \cap V$ there is a unique irreducible component 
$Z_{s i}^{\prime}$ of $Z_{s, \text { red }}$ such that $Z_{s i}^{\prime} \cap V=Z_{s i}(V)$. (For $s=o$ we may assume that $Z_{o} \cap V$ is connected and the corresponding component is $Z_{o}^{\prime}$. Hence the notations are compatible.) Let $k=\operatorname{dim}\left(Z_{s} \cap V\right)$ and $n=\operatorname{dim} X_{s}$, both of which we may assume to be independent of $s \in \gamma(V)$ in view of the smoothness of $f$ and $\gamma$ near $Z_{d_{o}}$. For $z \in Z$ let $b(z)=\operatorname{dim}_{z} \bar{\pi}^{-1} \bar{\pi}(z)$ and $b_{s i}(z)=\left.b(z)\right|_{Z_{s i}^{\prime}}$. Since $b(z)$ is upper semicontinuous with respect to the Zariski topology and $Z_{s i}^{\prime}$ is irreducible, there is a dense Zariski open subset $W_{s i}^{\prime}$ of $Z_{s i}^{\prime}$ such that $b_{s i}(z)$ has the minimum value, say $b_{s i}$, on $W_{s i}$. Then if $s$ is sufficiently near to $o$, we have $b_{s i} \leqq b_{o}, k-b_{s i}$ $=\operatorname{dim} \bar{\pi}\left(Z_{s i}^{\prime}\right) \leqq n$ and further $k-b_{o}=n$ since $\bar{\pi}\left(Z_{o}^{\prime}\right)=X_{o}$ by our assumption. It follows that $\operatorname{dim} \bar{\pi}\left(Z_{s i}^{\prime}\right)=n$, i.e., $\bar{\pi}_{s i}=\left.\bar{\pi}\right|_{Z^{\prime}}$, is surjective for all $s \in \gamma(V)$ since $X_{s}$ is irreducible if $V$ is sufficiently small as we may assume. Hence the image under $\bar{\pi}$ of $Z$ contains the open set $f^{-1} \gamma(V)$ and hence $\bar{\pi}$ is surjective by the irreducibility of $X$ and the properness of $\bar{\pi}$.

Next, let $F=\rho \times{ }_{s} \bar{\pi}: Z \rightarrow D \times{ }_{s} X, \bar{Z}=F(Z)$ and let $\rho_{1}: \bar{Z} \rightarrow D$ and $\pi_{1}: \bar{Z} \rightarrow X$ the natural projections. Let $A=\left\{z \in Z ; \operatorname{dim}_{z} F^{-1} F(z) \geqq 1\right\}$ and $\bar{A}=\rho_{1}(A)$. Then by Remmert $A$ and $\bar{A}$ are analytic subsets of $Z$ and $D$ respectively. On the other hand, since $Z_{d_{o}}$ is irreducible, from our assumption that $\operatorname{dim} \bar{\pi}\left(Z_{d_{o}}\right)=1$ it follows that $d_{o} \notin \bar{A}$ and therefore $\bar{A}$ is a proper analytic subset of $D$. This then implies that every fiber of $\rho_{1}$ is again of dimension 1 as well as $\rho$. Hence for every $d \in D, \operatorname{dim} \bar{\pi}\left(Z_{d}\right)=\operatorname{dim} \pi_{1}\left(\bar{Z}_{d}\right)=1$. Since $\bar{\pi}=\pi_{1} F$ and $\bar{\pi}(Z)=X$, we have $\pi_{1}\left(\bar{Z}_{s}\right)=X_{s}$ for every $s \in S$. Hence for every irreducible component $X_{s, k}$ of $X_{s, \text { red }}$ there is an irreducible component $\bar{Z}_{s, k}$ of $\bar{Z}_{s, \text { red }}$ such that $\pi_{1}\left(\bar{Z}_{s, k}\right)=X_{s, k}$. Note that since fibers of $\rho_{1}$ are connected as well as $\rho$ the dimension of the general fiber of $\rho_{1, s, k}: \bar{Z}_{s, k} \rightarrow X_{s, k}$ cannot be zero, and hence each fiber of $\rho_{1, s, k}$ has dimension one. Then it suffices to take any irreducible component $Z_{s, k}$ of $Z_{s, \text { red }}$ which are mapped surjectively onto $\bar{Z}_{s, k}$ by $F$.

Q.E.D.

Now we shall prove Theorem 3.4. We first reduce the proof to the case where $f \in \mathscr{C} / S$ : Suppose that the theorem is true if $f \in \mathscr{C} / S$. In general let $U$ be any dense Zariski open subset of $S$ such that $f_{U}: X_{U} \rightarrow U$ is smooth. Let $V=\left\{u \in U ; X_{u}\right.$ is uniruled $\}$. Then in view of the definition of loc- $\mathscr{C} / S$ together with our assumption it follows immediately that $V$ is open and closed in $U$. Hence $V=U$ since $o \in V$. Then since $U$ is dense in $S$, again by the definition of loc- $\mathscr{C} / S$ the theorem follows from the case $f \in \mathscr{C} / S$. So in what follows we assume that $f \in \mathscr{C} \mid S$.

Set $Y=\mathbb{P}^{1} \times X$ and let $h=p f: Y \rightarrow S$ be as in Section 1, a). Since $X_{o}$ is 
uniruled, by Lemma 2.2 there exists an irreducible component $D_{o, \alpha}$ of $D_{Y_{o} \text {,red }}$ such that $D_{o, \alpha} \cap H_{0} \neq \emptyset$ and that the natural morphism $\pi_{o, \alpha}: Z_{o, \alpha} \rightarrow X_{o}$ is surjective where $Z_{o, \alpha}=Z_{Y_{o}} \times_{D_{Y_{0}}} D_{o, \alpha}$ and $\pi_{o, \alpha}$ is the restriction of $\pi_{Y, o}$ to $Z_{o, \alpha}$. Identifying $D_{Y_{o}}$ with $D_{Y / S, o}$ as in Section 1, a) we regard $D_{o, \alpha}$ as an irreducible component of $\left(D_{Y / S, o}\right)_{\text {red }}$. Then take any irreducible component $D_{\alpha}$ of $D_{Y / S \text {,red }}$ which contains $D_{o, \alpha}$. Let $\beta_{\alpha}: D_{\alpha} \rightarrow S$ be the natural morphism. Let $U_{\alpha}=D_{o, \alpha} \cap H_{0}$ and $U_{\alpha}^{\prime}$ the set of smooth points of $U_{\alpha}$.

Lemma 2.6. There exists a Zariski open subset $V$ of $D_{o, \alpha}$ with $V \subseteq U_{\alpha}^{\prime}$ such that $\beta_{\alpha}$ is smooth at each point of $V$.

Proof. Let $n=\operatorname{dim} X_{o}$. Let $W$ be a nonempty Zariski open subset of $Z_{o, \alpha}$ on which $\pi_{o, \alpha}$ is smooth. This is possible since $\pi_{o, \alpha}$ is surjective. Set $V=U_{\alpha}^{\prime} \cap \rho_{o, \alpha}(W)$. Then it is immediate to see that $V$ is Zariski open in $D_{o, \alpha}$. We show that this $V$ has the desired property. Let $d \in V$ be any point. Then $\pi_{o, \alpha}$ is smooth at points of $Z_{d} \cap W \neq \emptyset$ where $Z_{d}=Z_{o, d}=\left(Z_{o, \alpha}\right)_{d}$. Moreover we see readily that there exist a neighborhood $V(d)$ of $d$ in $V$ and an irreducible and smooth subspace $N$ of $V(d)$ of dimension $n-1$ with $d \in N$ such that if we put $M=\rho_{o, \alpha}^{-1}(N)$, then $h=\left.\pi_{o, \alpha}\right|_{M}: M \rightarrow X_{o}$ is locally biholomorphic at some point of $Z_{d}$.

Now in general let $\Theta_{B}$ be the sheaf of germs of holomorphic vector fields on $B$. Then we have the natural homomorphism of $\mathcal{O}_{M}$-modules $\lambda: \Theta_{M} \rightarrow h^{*} \Theta_{X_{0}}$. Since $h$ is locally biholomorphic at a point of $Z_{d}, \lambda$ is injective and, after restricting to $C=Z_{d}$, we obtain the following exact sequence of $\mathcal{O}_{C}$-modules

$$
\left.\left.0 \longrightarrow \Theta_{M}\right|_{C} \longrightarrow h^{*} \Theta_{X_{0}}\right|_{C} \longrightarrow 2 \longrightarrow 0
$$

where 2 is a torsion $\mathcal{O}_{C}$-module. Since $H^{1}(C, 2)=0$ and $\left.h^{*} \Theta_{X_{0}}\right|_{C}=h_{d}^{*} \Theta_{X_{0}}$ where $h_{d}$ is the holomorphic map $\mathbb{P}^{1} \rightarrow X_{o}$ corresponding to $d \in U_{\alpha}$, for the lemma we have only to show that $H^{1}\left(M,\left.\Theta_{M}\right|_{C}\right)=0$, in view of Section 1, c). On the other hand, since the normal bundle of $C$ in $M$ is trivial, we have the following exact sequence of $\mathcal{O}_{C}$-modules

$$
\left.0 \longrightarrow \Theta_{C} \longrightarrow \Theta_{M}\right|_{C} \longrightarrow \mathcal{O}_{C}^{\oplus(n-1)} \longrightarrow 0 \text {. }
$$

Then from the long exact sequence associated to this sequence we get the desired vanishing of $H^{1}\left(C,\left.\Theta_{M}\right|_{C}\right)$ since $C \cong \boldsymbol{P}^{1}$.

Q.E.D.

Remark 2.7. Tensoring $\mathcal{O}_{C}(-1)$ with the two short exact sequences appearing in the above proof and taking the long exact sequences of cohomology 
we even cbtain the vanishing: $H^{1}\left(C, h_{d}^{*} \Theta_{X_{o}}(-1)\right)=0$. In fact this also has some geometric significance which will be discussed at the end of this section.

Now fix any relatively compact subdomain $S^{\prime} \ni o$. It suffices to show the theorem for $f_{S^{\prime}}: X_{S^{\prime}} \rightarrow S^{\prime}$ since $S^{\prime}$ is arbitrary. For notational simplicity we denote $S^{\prime}$ again by $S$ and hence $X_{S^{\prime}}$ by $X$ and $f_{S^{\prime}}$ by $f$. We then consider the following commutative diagram

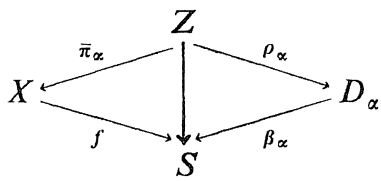

and show that this satisfies the conditions of Lemma 2.5. First of all by our restriction of $S$ as above, $\beta_{\alpha}$ and $\bar{\pi}_{\alpha}$ are proper (cf. Section 1, d)). Further by our definition $\rho_{\alpha}$ is flat and surjective, and in a neighborhood of $X_{o}, f$ is smooth and has connected fibers by assumption. We take $d_{o} \in D_{\alpha, o}$ to be any point from $V \cong U_{\alpha}$ in Lemma 2.6. Then $\beta_{\alpha}$ is smooth at $d_{o}$, so that 1 ) of Lemma 2.5 is satisfied. Moreover since $\left.d_{o} \in U_{\alpha}, 2\right)$ also is satisfied in view of Section 1, b). Finally taking $\left.Z_{o}^{\prime}=Z_{o, \alpha}, 3\right)$ also is true. Thus by that lemma for every $s \in S$ and for every irreducible component $X_{s, k}$ of $X_{s, \text { red }}$ there exists an irreducible component $Z_{\alpha, s}^{k}$ of $\left(Z_{\alpha, s}\right)_{\text {red }}$ such that $\bar{\pi}_{\alpha}\left(Z_{\alpha, s}^{k}\right)=X_{s, k}$ and $\operatorname{dim} \bar{\pi}_{\alpha}\left(\left(Z_{\alpha, s}^{k}\right)_{d}\right)=1$ for all $d \in D_{\alpha, s}=\rho_{\alpha}\left(Z_{\alpha, s}^{k}\right)$. Let $\mu: \widetilde{Z}_{\alpha, s}^{k} \rightarrow Z_{\alpha, s}^{k}$ be the normalization of $Z_{\alpha, s}^{k}$ and $\tilde{g}_{s}^{k}: \widetilde{Z}_{\alpha, s}^{h}$ $\rightarrow D_{\alpha, s}^{k}$ be $\mu$ followed by the natural map $\rho_{\alpha, s}^{k}: Z_{\alpha, s}^{k} \rightarrow D_{\alpha, s^{*}}^{k}$ Let $g_{s}^{k}: \widetilde{Z}_{\alpha, s}^{k} \rightarrow T_{\alpha, s}^{k}$, $T_{\alpha, s}^{k} \rightarrow D_{\alpha, s}^{k}$ be the Stein factorization of $\tilde{g}_{s}^{k}$. Then $g_{s}^{k}$ is a proper morphism of irreducible complex spaces with connected fibers. Further, every fiber of $\rho_{\alpha}$, and hence that of $\rho_{\alpha, s}^{k}$ also, is union of rational curves as a specialization of $\mathbb{P}^{1}$. Hence the general fiber of $g_{s}^{k}$ is isomorphic to $\mathbb{P}^{1}$ being a normalization of that of $\rho_{\alpha, s^{*}}^{k}$. Therefore $g_{s}^{k}$ together with $\bar{\pi}_{\alpha} \mu: \widetilde{Z}_{\alpha, s}^{k} \rightarrow X_{s, k}$ gives a covering family of rational curves on $X_{s, k}$. Hence $X_{s, k}$ is uniruled by Lemma 2.2. $\quad$ Q.E.D.

In the rest of this section we shall give a geometric implication of the vanishing result mentioned in Remark 2.7. Since the method of proof is essentially the same as above (cf. Mori [8]), we omit the proof and give the statements only.

Definition 2.8. Let $X$ be an irreducible compact complex space. We call $X$ rationally connected (resp. to a point $x \in X$ ) if there exists a covering family of rational curves $(g: Z \rightarrow T, \psi: Z \rightarrow X)$ on $X$ such that $\psi \times{ }_{T} \psi: Z \times{ }_{T} Z \rightarrow X \times X$ is surjective (resp. $g \psi^{-1}(x)=T$ ). 
Remark. It is immediate to see that if $X$ is rationally connected, then it is rationally connected to any general point of $X$. Further, $X$ is rationally connected if and only if there exists a Zariski open subset $V \cong X$ such that any two points $x_{1}, x_{2} \in V$ can be connected by an irreducible rational curve. In this case $X$ is Moishezon. If, further, $X$ is normal, then $X$ is simply connected. The proof of the last two facts will be given in the Appendix.

Proposition 2.9. Let $f: X \rightarrow Y$ be a proper smooth morphism of irreducible complex spaces with connected fibers. Suppose that $f \in \mathscr{C} / S$ and $X_{o}$ is rationally connected to a point $x \in X_{o}$ for some $o \in S$. Then there exists a dense open subset $o \in U \subseteq S$ such that $X_{s}$ is rationally connected for every $s \in U$.

Corollary. A compact complex manifold $X$ which is rationally connected to some of its points is rationally connected.

Corollary is false if $X$ has a singularity. (Consider the projective cone over a non-uniruled projective variety.) The above proposition shows that general quartic threefolds in $\boldsymbol{P}^{4}$ are rationally connected in view of the result of Segre-Iskovskih-Manin.

\section{§3. Locus of Ruled Manifolds}

We begin with the following lemma analogous to Lemma 2.2.

Lemma 3.1. Let $X$ be an irreducible compact Moishezon space of dimension $n$. Then the following conditions are equivalent. 1) $X$ is ruled. 2) Let $Y=\boldsymbol{P}^{1} \times X$. Then there exists an irreducible complex subspace $B$ of $D_{Y, \text { red }}$ of dimension $n-1$ such that a) $B \cap H_{0} \neq \emptyset$ (cf. Section $\left.1, b\right)$ ) and b) $\pi_{B}: Z_{B} \rightarrow X$ is bimeromorphic, where $Z_{B}=Z_{Y} \times_{D_{Y}} B$ and $\pi_{B}=\left.\pi_{Y}\right|_{Z_{B}}$.

Proof. 1) $\rightarrow$ 2). Let $\bar{\varepsilon}: \boldsymbol{P}(E) \rightarrow N$ be a $\boldsymbol{P}^{1}$-bundle as in 1) of Definition 2.1. Since $N$ is Moishezon, $\bar{\varepsilon}$ is actually bimeromorphic to a product bundle $N \times \boldsymbol{P}^{1} \rightarrow N$. Therefore we may assume that $\boldsymbol{P}(E)=N \times \boldsymbol{P}^{1}$. Let $\lambda: N \times \boldsymbol{P}^{1} \rightarrow X$ be the given bimeromorphic map. Then there exists a Zariski open subset $U \subseteq N$ such that $\lambda$ is holomorphic on $U \times \boldsymbol{P}^{1}$. Let $\Gamma \leqq U \times \boldsymbol{P}^{1} \times X$ be the graph of this holomorphic map;

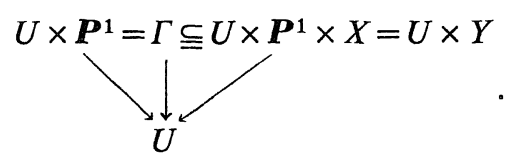


Considering the above diagram as a smooth family of subspaces of $Y$ parametrized by $U$ we get a holomorphic map $\tau: U \rightarrow D_{Y}$ such that the family is induced from the corresponding universal family. Let $B$ be the closure of $\tau(U)$ in $D_{Y}$, which is an analytic subset of $D_{Y}$ (cf. [1]). We shall see that this $B$ satisfies all the desired properties. In fact we have the following commutative diagram of meromorphic maps

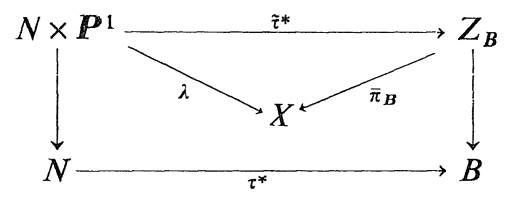

where $\tau^{*}$ is the meromorphic map extending $\tau$ and $\tilde{\tau}^{*}$ is induced by $\tau^{*}$. Since $\tilde{\tau}^{*}$ is surjective, $\bar{\pi}_{B}$ must be bimeromorphic as well as $\lambda$. (This is b).) In particular $\operatorname{dim} Z_{B}=n$. Hence $\operatorname{dim} B=n-1$ since the general fiber of $\rho_{B}: Z_{B} \rightarrow B$ is $\boldsymbol{P}^{1}$. In fact, we have $H_{0} \cap B \supseteqq \tau(U) \neq \varnothing$, which is a).

2) $\rightarrow 1$ ). $\quad \varphi_{Y} \times \rho_{Y}$ induces a bimeromorphic morphism $Z_{B} \rightarrow \mathbb{P}^{1} \times B$. Thus $X$ is bimeromorphic to $\boldsymbol{P}^{1} \times B$ as desired.

Q. E.D.

We consider the following commutative diagram of irreducible complex spaces

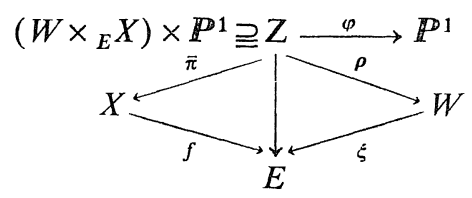

where $f$ is proper, smooth and has connected fibers, $\rho$ and $\xi$ are proper, flat and surjective, and where $\bar{\pi}, \rho$ and $\varphi$ are induced by the projections from $\left(W \times{ }_{E} X\right)$ $\times \boldsymbol{P}^{1}$ onto $X, W$ and $\boldsymbol{P}^{1}$ respectively.

Lemma 3.2. Suppose further that the following conditions are satisfied: 1) The general fiber of $\xi$ is irreducible, 2) $\varphi_{w_{o}}: Z_{w_{o}} \rightarrow \mathbb{P}^{1}$ is isomorphic for some $w_{o} \in W$, and 3) $\pi_{e_{0}}: Z_{e_{o}} \rightarrow X_{e_{o}}$ is bimeromorphic for some $e_{o} \in E$. Then $X_{e}$ is ruled for all $e \in E$.

Proof. $\alpha$ ) We show that $X_{e}$ is ruled for general $e \in E$. Define $U=\{w \in W$; $\varphi_{w}: Z_{w} \rightarrow P^{1}$ is isomorphic $\}$, and $M=\left\{e \in E ; \pi_{e}: Z_{e} \rightarrow X_{e}\right.$ is bimeromorphic $\}$. Then $U$ and $M$ are Zariski open in $W$ and $E$ respectively (cf. [1, Lemma 5.5]). Moreover since $w_{o} \in U$ and $e_{o} \in M$, they are nonempty. Let $\bar{U}=\xi(U)$. Then it is easy to see that $\bar{U}$ contains a Zariski open subset, say $V_{0}$, of $E$. Restricting $V_{0}$ if necessary, we may assume that $W_{e}$ are reduced and irreducible for all 
$e \in V_{0}$ by 1) and [1, Lemma 1.4]. Then we show that $X_{e}$ is ruled for any $e \in V$ $=V_{0} \cap M$. Since $X_{e}$ is bimeromorphic to $Z_{e}$, it is enough to show that $Z_{e}$ is ruled for any $e \in V_{0}$. First since $U \cap W_{e}$ is dense in $W_{e}$, the general fiber of $\rho_{e}: Z_{e} \rightarrow W_{e}$ is isomorphic to $\boldsymbol{P}^{1}$ via $\varphi_{e}$. Then it follows that $\rho_{e} \times \varphi_{e}: Z_{e} \rightarrow$ $W_{e} \times P^{1}$ is bimeromorphic (cf. Section $1, \mathrm{~b}$ )). Thus $Z_{e}$ is ruled. (By the flatness of $\rho, Z_{e}$ is actually reduced and irreducible.)

$\beta)$ Let $E^{\prime}=\left\{e \in E ; X_{e}\right.$ is not ruled $\}$. Supposing that $E^{\prime} \neq \emptyset$ we shall derive a contradiction. Let $H=\{t \in C ;|t|<1\}$. Take a morphism $q: H \rightarrow E$ in such a way that $q^{-1}(V)=H^{\prime}:=H-\{0\}$ and $q(0) \in E^{\prime}$. Then taking the base change of everything to $H$ by $q$ we may assume from the beginning that $E=H$, $E^{\prime}=\{0\}$ and $V=H^{\prime}$. (Recall that $f, \rho, \xi$ are all flat.) In particular we shall denote the point of $E$ by $t$. Recall from $\alpha)$ that for each $t \neq 0,\left(\rho_{t} \times \varphi_{t}\right) \bar{\pi}_{t}^{-1}$ gives a bimeromorphic map of $X_{t}$ and $W_{t} \times \boldsymbol{P}^{1}$. This in turn implies that $h=(\rho \times \varphi) \bar{\pi}^{-1}$ gives a bimeromorphic map of $X$ and $W \times \boldsymbol{P}^{1}$ over $E$. Let $r: \widetilde{W} \rightarrow W$ be any resolution of $W . \quad \widetilde{W}$ is a complex space over $E$ by $\xi r$. Let $\tilde{h}: X \rightarrow \widetilde{W} \times \boldsymbol{P}^{1}$ be the bimeromorphic map over $E$ induced by $h$. Now if $X_{0}$ corresponds to an irreducible component $\tilde{W}_{0}^{\prime}$ of $\tilde{W}_{0} \times \boldsymbol{P}^{1}$ by $\tilde{h}$, then $X_{0}$ is ruled since $\tilde{W}_{0}^{\prime}$ is necessarily of the form $\tilde{W}_{0 i} \times \boldsymbol{P}^{1}$ for some irreducible component of $\widetilde{W}_{0 i}$ of $\tilde{W}_{0}$. This is impossible by our assumption that $0 \in E^{\prime}$. Thus $X_{0}$ must be an exceptional divisor for the map $h$, i.e., must correspond to a lower dimensional subspace of $\tilde{W} \times \boldsymbol{P}^{1}$. Then since $\tilde{W} \times \boldsymbol{P}^{1}$ is nonsingular, $X_{0}$ is again ruled (cf. [4, Lemma 4.1]). Thus we get a desired contradiction.

Q.E.D.

Proposition 3.3. Let $f: X \rightarrow S$ be a proper smooth morphism of irreducible complex spaces with connected fibers. Let $R=\left\{s \in S ; X_{s}\right.$ is ruled $\}$. Suppose that $f$ is Moishezon (cf. Section $1, d)$ ). Then for any relatively compact open subset $S^{\prime}$ of $S, R \cap S^{\prime}$ is a union of at most countably many analytic subsets of $S^{\prime}$.

Proof. Fix any relatively compact open subset $S^{\prime} \subseteq S$. Replacing $S$ by $S^{\prime}$ we denote $S^{\prime}$ again by $S$. Let $n=\operatorname{dim} X_{s}$, which is independent of $s$ since $f$ is smooth and $X$ and $S$ are irreducible. We first construct the following commutative diagram of irreducible complex spaces

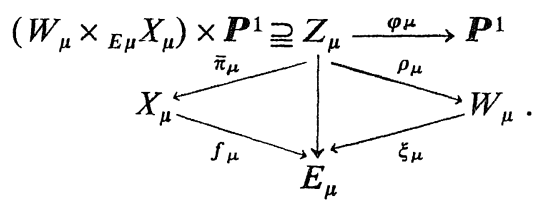


Fix any irreducible component $D_{\alpha}$ of $D_{Y / S \text {,red }}$ where $Y=X \times \mathbb{P}^{1}$. Let $E_{\mu}$ be any irreducible component of $D_{D_{\alpha} / S \text {,red }}$. Let $\xi_{\mu}: W_{\mu} \rightarrow E_{\mu}$ be the morphism induced from the universal family $\rho_{D_{\alpha} / S}: W_{D_{\alpha} / S} \rightarrow D_{D_{\alpha} / S}$ by restriction so that we have the following commutative diagram corresponding to the one in Section 1, a)

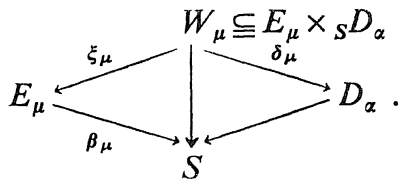

Let $Z_{\mu}=W_{\mu} \times{ }_{D_{\alpha}} Z_{\alpha}$ and let $\rho_{\mu}: Z_{\mu} \rightarrow W_{\mu}$ be induced from $\rho_{\alpha}: Z_{\alpha} \rightarrow D_{\alpha}$. Let $X_{\mu}=E_{\mu} \times{ }_{S} X$ and let $f_{\mu}: X_{\mu} \rightarrow E_{\mu}$ be induced from $f$. On the other hand, $\pi_{\alpha}: Z_{\alpha}$ $\rightarrow X$ (resp. $\varphi_{\alpha}: Z_{\alpha} \rightarrow \mathbb{P}^{1}$ ) (cf. Section 1, a)) induces the morphism $\bar{\pi}_{\mu}: Z_{\mu} \rightarrow X_{\mu}$ (resp. $\left.\varphi_{\mu}: Z_{\mu} \rightarrow \mathbb{P}^{1}\right)$, and the natural inclusion $Z_{\alpha} \subseteq\left(D_{\alpha} \times{ }_{S} X\right) \times \mathbb{P}^{1}$ induces the natural inclusion $Z_{\mu} \subseteq\left(W_{\mu} \times{ }_{S} X\right) \times \mathbb{P}^{1}=\left(W_{\mu} \times E_{\mu} X_{\mu}\right) \times \mathbb{P}^{1}$ such that $\pi_{\mu}, \rho_{\mu}$ and $\varphi_{\mu}$ are induced by the natural projections from $\left(W_{\mu} \times E_{E_{\mu}} X_{\mu}\right) \times \mathbb{P}^{1}$ onto the corresponding factors. This completes the construction of $(*)$. Now we impose some additional conditions on our choice of $D_{\alpha}$ and $E_{\mu}$, so that (*) satisfies the conditions of Lemma 3.2. 1) There exists a dense Zariski open subset $V_{\mu}$ of $E_{\mu}$ such that $W_{\mu, e}$ are reduced and irreducible of dimension $n-1$ for all $e \in V_{\mu}$. 2) Let $U_{\alpha}=\left\{d \in D_{\alpha} ; \varphi_{\alpha, d}: Z_{\alpha, d} \rightarrow \mathbb{P}^{1}\right.$ is isomorphic $\}$. Then $\delta_{\mu}\left(W_{\mu}\right) \cap U_{\alpha} \neq \emptyset$. 3) $\bar{\pi}_{\mu, e^{\prime}}: Z_{\mu, e^{\prime}} \rightarrow X_{\mu, e^{\prime}}$ is a bimeromorphic morphism for some $e^{\prime} \in E_{\mu}$. Note that from 2) it follows that $W_{\mu}$ is reduced and irreducible and from 3) that $Z_{\mu, e}$ is irreducible as well as $X_{\mu, e}$. Then we see readily that $(*)$ satisfies all the conditions of Lemma 3.2. Therefore by that lemma it follows that $X_{\mu, e}=X_{\beta_{\mu}(e)}$ is ruled for all $e \in E$.

Now let $S_{\mu}=\beta_{\mu}\left(E_{\mu}\right)$. We show that $S_{\mu}$ is an analytic subset of $S$. In fact, since $f$ is Moishezon, $D_{\alpha} \rightarrow S$ is again proper and Moishezon (cf. Section 1, d)). Hence $E_{\mu} \rightarrow S$ also is proper and Moishezon. Thus by Remmert $S_{\mu}$ is an analytic subset of $S$. Let $\mathfrak{M}$ be the set of indices $(\alpha, \mu)$ such that the pair $\left(D_{\alpha}, E_{\mu}\right)$ satisfies the conditions 1)-3) above. Then by [2] $\mathfrak{A}$ is at most a countable set. We have thus constructed at most countably many analytic subsets $S_{\mu}=S_{\alpha, \mu}$, $(\alpha, \mu) \in \mathfrak{U}$, of $S$ such that $\cup_{(\alpha, \mu) \in \mathfrak{Q}} S_{\alpha, \mu} \subseteq R$.

It remains to show that $R \cong \cup_{\mathfrak{U}} S_{\alpha, \mu}$ for some $(\alpha, \mu) \in \mathfrak{A}$. Namely we have to show that if $X_{s}$ is ruled for some $s \in S$, then $s \in S_{\alpha, \mu}$ for some $(\alpha, \mu) \in \mathfrak{A}$. Let $Y_{s}=\boldsymbol{P}^{1} \times X_{s}$. Then by Lemma 3.1 there exists an irreducible analytic subset $B_{s}$ of $D_{Y_{s}}$ of dimension $n-1$ such that $B_{s} \cap H_{0} \neq \emptyset$ and that $\pi_{B_{s}}: Z_{B_{s}} \rightarrow X_{s}$ is 
bimeromorphic (cf. Section 1, b)). Identifying $D_{Y_{s}}$ with $D_{Y / S, s}$, let $D_{\alpha}$ be any irreducible component of $D_{Y / S \text {,red }}$ containing $B_{s}$. Then $B_{s}$ defines a point $e_{s}$ of $D_{D_{\alpha} / S, s}$. Let $E_{\mu}$ be any irreducible component of $D_{D_{\alpha} / S \text {,red }}$ containing $e_{s}$. Then we claim that the pair $\left(D_{\alpha}, E_{\mu}\right)$ satisfies 1$\left.)-3\right)$, i.e., $(\alpha, \mu) \in \mathfrak{A}$. This would prove the proposition since $s=\beta_{\mu}\left(e_{s}\right) \in \beta_{\mu}\left(E_{\mu}\right)=S_{\alpha, \mu}$. Now we prove 1)-3). 1) $\delta_{\mu}\left(W_{\mu}\right) \cap U_{\alpha} \supseteqq B_{s} \cap U_{\alpha}=B_{s} \cap H_{0} \neq \emptyset$. 2) $W_{\mu, e_{s}}=B_{s}$ and hence is reduced, irreducible and of dimension $n-1$. By the flatness of $\xi_{\mu}$ it follows readily that the same is true for any $W_{\mu, e^{\prime}}$ where $e^{\prime}$ is from some Zariski open subset of $E_{\mu}$ (cf. [1, Lemma 1.4]). 3) It suffices to take $e=e_{s}$ by our construction. Q. E. D.

Proposition 3.4. Let $f: X \rightarrow S$ be a proper smooth morphism of irreducible complex spaces with connected fibers. Suppose that there exist compact complex spaces $X^{*}$ and $S^{*}$ such that 1) $X$ and $S$ are Zariski open subsets of $X^{*}$ and $S^{*}$ respectively, and 2) $f$ extends to a Moishezon morphism $f^{*}: X^{*} \rightarrow S^{*}$. Then the set $R=\left\{s \in S ; X_{s}\right.$ is ruled $\}$ is a union of at most countably many analytic subsets of $S$ which extend to analytic subsets of $S^{*}$.

The proof is the same as that of the above proposition if we take $D_{\alpha}$ to be irreducible components of $D_{X^{*} / S^{*}}$ instead of those of $D_{X / S}$.

\section{Appendix}

Let $X$ be an irreducible compact complex space. Suppose that $X$ is rationally connected to $x \in X$ (cf. Definition 2.8). First we show that $X$ is Moishezon. In fact, in the notation of Definition $2.8 \psi^{-1}(x)$ is Moishezon by [3] and hence $T$ also is Moishezon. Then since $g: Z \rightarrow T$ is a Moishezon morphism, being of fiber dimension $1, Z$ also is Moishezon. Hence $X$ is Moishezon as a surjective image of $Z$. Next we show the following:

Proposition A. Suppose that $X$ is normal. Then $X$ is simply connected.

Proof. Let $\alpha: \tilde{X} \rightarrow X$ be the universal covering of $X$. Suppose that there exist an unramified covering $\alpha_{1}: \tilde{X}_{1} \rightarrow X_{1}$ of complex spaces and surjective morphisms $\varphi: X_{1} \rightarrow X$ and $\tilde{\varphi}: \tilde{X}_{1} \rightarrow \tilde{X}$ such that $\varphi \alpha_{1}=\alpha \tilde{\varphi}$ and that $\left(\varphi \alpha_{1}\right)^{-1}(x)$ is connected. Then it follows that $\tilde{\varphi}^{-1}\left(\alpha^{-1}(x)\right)$, and hence $\alpha^{-1}(x)$ also, is connected. This implies that $\alpha$ is isomorphic and hence $X$ is simply connected. Thus it suffices to show the existence of $\alpha_{1}, \varphi, \tilde{\varphi}$ as above. By our assumption it follows that there exists an irreducible component $D_{\alpha}$ of $D_{X, \text { red }}$ such that 
$Z_{\alpha, d}=\rho_{\alpha}^{-1}(d)$ is an irreducible and rational curve, and that $D_{\alpha} \times\{x\} \subseteq Z_{\alpha}$. Then as in the proof of Lemma 2.2 after a suitable base change $T \rightarrow D_{\alpha}$ which is proper and surjective, the induced map $Z_{T}=Z_{\alpha, T} \rightarrow T$ is bimeromorphic to the projection $T \times \mathbb{P}^{1} \rightarrow T$. In particular if we take $T$ to be nonsingular as we may, then for any resolution $Z \rightarrow Z_{T}$ of $Z_{T}$ the natural map $Z \rightarrow T$ induces an isomorphism $\pi_{1}(Z) \cong \pi_{1}(T)$ of the fundamental groups. Then we set $X_{1}=Z_{T}$ and define $\varphi$ by the natural morphism $Z_{T} \rightarrow X$ which is obviously surjective. Next we construct $\alpha_{1}$. Let $\tilde{Z}$ be an irreducible component of $Z \times_{X} \tilde{X}$. Let $\tilde{X}_{1}$ be the image of $Z$ under the natural proper bimeromorphic morphism $Z \times_{X} \tilde{X} \rightarrow$ $X_{1} \times{ }_{X} \tilde{X}$. Then $\tilde{X}_{1}$ is an irreducible component of $X_{1} \times_{X} \tilde{X}$. Let $\alpha_{1}: \tilde{X}_{1} \rightarrow X_{1}$ be the natural projection $\tilde{Z} \rightarrow Z$. We show that the other projection $\tilde{\varphi}: \tilde{X}_{1} \rightarrow \tilde{X}$ is surjective. In fact, $\tilde{\varphi}$ is proper as well as $\varphi$. Therefore $\tilde{\varphi}\left(\tilde{X}_{1}\right)$ is an analytic subset of $\tilde{X}$. On the other hand, we have $\operatorname{dim} \tilde{\varphi}\left(\tilde{X}_{1}\right)=\operatorname{dim} \tilde{X}$. Since $\tilde{X}$ is normal as well as $X$, this implies that $\tilde{\varphi}\left(\tilde{X}_{1}\right)=\tilde{X}$. Thus it remains only to show that $\left(\varphi \alpha_{1}\right)^{-1}(x)$ is connected. First, since $\pi_{1}(Z) \cong \pi_{1}(T)$, there exists an unramified covering $u: \tilde{T} \rightarrow T$ such that $\tilde{Z}=Z \times_{T} \tilde{T}$. Let $X_{1}^{\prime}=X_{1} \times_{T} \widetilde{T}$ and let $\alpha_{1}^{\prime}: X_{1}^{\prime} \rightarrow X_{1}$ be the natural projection. $X_{1}^{\prime}$ is naturally bimeromorphic to $Z$. Then we get the following commutative diagram

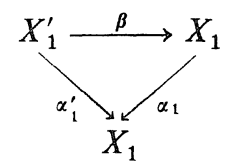

where $\beta$ is a bimeromorphic map. Since $\alpha_{1}$ and $\alpha_{1}^{\prime}$ are unramified, it is easy to show that $\beta$ is actually isomorphic. Then $\left(\varphi \alpha_{1}\right)^{-1}(x)=\alpha_{1}^{\prime-1}(T \times\{x\})=\widetilde{T} \times\{x\}$, which is connected.

Q.E.D.

Note finally that there is no holomorphic $p$-form on $X$ for any $p>0$ by Roytman $[9]^{1)}$. (In fact, in our special case the proof becomes quite simple.)

\section{References}

[1] Fujiki, A., Closedness of the Douady spaces of compact Kähler spaces, Publ. RIMS, Kyoto Univ., 14 (1978), 1-52.

[2] - Countability of the Douady space of a complex space, Japan J. Math., 5 (1979), 431-447.

[3] - On the Douady space of a compact complex space in the category $\mathscr{C}$, to appear in Nagoya J. Math., 85 (1982).

1) This was kindly pointed out to the author by Prof. Sumihiro. 
[4] — A theorem on bimeromorphic maps of Kähler manifolds and its applications, Publ. RIMS, Kyoto Univ., 17 (1981), 735-754.

[5] — Coarse moduli space for polarized compact Kähler manifolds and polarized algebraic manifolds, to appear.

[6] Relative algebraic reduction and relative Albanese map for a fiber space in $C$, to appear.

[7] Grothendieck, A., Technique de construction et théorèmes d'existence en géométrie algébrique IV, Séminaire Bourbaki, ${ }^{\circ} 221,1960 / 61$.

[ 8 ] Mori, S., Projective manifolds with ample tangent bundles, Ann. of Math., 110 (1979), 593-606.

[9] Roytman, A. A., On $\Gamma$-equivalence of zero-dimensional cycles, Math. USSR Sbornik, 15 (1971), 555-567.

Added in proof: After submitting this paper the author received a preprint: Levine, M., Deformations of uni-ruled varieties, which contains essentially the same result as the present paper. 